\title{
Antimicrobial Susceptibility and Methicillin Resistance in Staphylococcus pseudintermedius and Staphylococcus schleiferi subsp. coagulans Isolated from Dogs with Pyoderma in Japan
}

\author{
Tetsuji KAWAKAMI ${ }^{1}$, Sanae SHIBATA ${ }^{1)}$, Nobuo MURAYAMA ${ }^{2)}$, Masahiko NAGATA ${ }^{2)}$, Koji NISHIFUJI ${ }^{3)}$, \\ Toshiroh IWASAKI ${ }^{3)}$ and Tsuneo FUKATA ${ }^{1) *}$ \\ ${ }^{1)}$ The United Graduate School of Veterinary Sciences, Gifu University, 1-1 Yanagido, Gifu 501-1193, ')ASC Dermatology Service, 1-3-2 \\ Jindaijihigashi, Chofu, Tokyo 180-0012 and ${ }^{3}$ Department of Veterinary Medicine, Tokyo University of Agriculture and Technology, 3- \\ 8-1 Harumi-cho, Fuchu, Tokyo 183-8538, Japan
}

(Received 21 April 2010/Accepted 27 July 2010/Published online in J-STAGE 10 August 2010)

ABSTRACT. To understand species distribution, trends of antimicrobial susceptibility and prevalence of methicillin resistance in canine staphylococci in Japan, 190 coagulase-positive staphylococci (CoPS) were isolated from dogs with pyoderma in 2 Japanese veterinary referral hospitals. Using a multiplex polymerase chain reaction (M-PCR) method, two CoPS species were identified: 170 Staphylococcus pseudintermedius $(89.5 \%)$ and $20 \mathrm{~S}$. schleiferi subsp. coagulans isolates $(10.5 \%)$. In these isolates, susceptibility to 7 antimicrobial agents was determined. Overall, the levels of susceptibility to cefalexin (CEX), amoxicillin/clavulanic acid (CVA/AMPC), minocycline (MINO), ofloxacin (OFLX), norfloxacin (NFLX), lincomycin (LCM) and clindamycin (CLDM) in S. pseudintermedius isolates were $38.2,52.4,34.7,31.2,34.1,1.2$ and $11.2 \%$, respectively. In $S$. schleiferi subsp. coagulans isolates, $55 \%$ demonstrated susceptibility to CEX, $80 \%$ to CVA/AMPC, $70 \%$ to MINO, $45 \%$ to OFLX or NFLX and $30 \%$ to CLDM. None of S. schleiferi subsp. coagulans isolates was susceptible to LCM. To determine the prevalence of methicillin-resistant strains, we used a PCR method, which enabled detection of the fragment of mecA gene in 66.5\% (113 of 170) in S. pseudintermedius and 30.0\% (6 of 20) in S. schleiferi subsp. coagulans isolates. The frequencies of susceptibility to CEX, CVA/AMPC, OFLX, NFLX and CLDM were significantly lower in methicillin-resistant CoPS than in methicillin-susceptible CoPS isolates. These data suggest a high level of methicillin resistance in staphylococci isolated from dogs with pyoderma in Japan.

KEY WORDS: antimicrobial susceptibility, canine pyoderma, methicillin-resistant staphylococci, minocycline, Staphylococcus species.

J. Vet. Med. Sci. 72(12): 1615-1619, 2010

Pyoderma is a common infectious skin disorder in dogs, and most of these cases are caused by staphylococcal strains [15]. Among them, several coagulase-positive staphylococcal (CoPS) species such as Staphylococcus intermedius, $S$. schleiferi and $S$. aureus have been isolated from the skin in canine pyoderma $[3,12,22]$. In particular, $S$. intermedius has been recognized to constitute normal skin flora and to occasionally cause a variety of infections such as pyoderma in dogs $[4,24]$. However, recent reports indicated that phenotypically identified $S$. intermedius strains could include not only true $S$. intermedius strains but also novel CoPS species such as $S$. pseudintermedius and $S$. delphini, and in fact, most of them were identified as $S$. pseudintermedius strains, not $S$. intermedius [1, 31].

The treatment of canine pyoderma usually involves antimicrobial therapy. Above all, cefalexin has been recommended as the first choice in canine pyoderma therapy [25]. However, previous studies showed that multidrug-resistant and methicillin-resistant staphylococci are present in increasing frequencies in dogs $[16,22,23,30,41]$. These strains cause therapeutic failure in veterinary practices and, potentially, zoonotic problems [38]. However, there are few

\footnotetext{
* Correspondence to: Fukata, T., The United Graduate School of Veterinary Sciences, Gifu University, 1-1 Yanagido, Gifu 5011193, Japan.

e-mail: tfukata@gifu-u.ac.jp
}

recent publications concerning antimicrobial susceptibility and methicillin resistance in CoPS isolated from canine pyoderma in Japan.

The purpose of this study was to determine the frequency of antimicrobial susceptibility and prevalence of methicillin resistance in staphylococcal isolates, and to demonstrate the antimicrobials that are available to treat methicillin-resistant CoPS isolates from dogs with pyoderma in Japan.

\section{MATERIALS AND METHODS}

Bacterial isolates: Bacterial isolates from lesional skin of 186 clinical cases diagnosed with canine pyoderma were collected between June 2007 and April 2009 in ASC Dermatology Service (Tokyo, Japan) and Tokyo University of Agriculture and Technology, Animal Medical Center (Tokyo, Japan). A sterile BBL CultureSwab (Becton, Dickinson and Co., Franklin Lakes, NJ, U.S.A.) was used to sample from the skin. All swabs were transported to our laboratory, following placement in Stuart's transport medium (Becton, Dickinson and Co.). The methods to identify CoPS isolates were performed as previously described $[10,14]$ with partially modification. Swabs were inoculated by streaking onto mannitol salt agar plates (Nissui Pharmaceutical Co., Ltd., Tokyo, Japan). A single representative colony of each sample was isolated and pure cultured on 
nutrient agar plate (Nissui Pharmaceutical Co., Ltd.). Gram-positive cocci were tested with 3\% hydrogen peroxide (Kanto Chemical Co., Inc., Tokyo, Japan) for catalase activity. Catalase-positive cocci were mixed with rabbit plasma (Kohjin Bio Co., Ltd., Saitama, Japan) and incubated overnight to test for coagulase production. Coagulase-positive isolates were stored in Microbank ${ }^{\circledR}$ (Pro-Lab Diagnostics Inc., Richmond Hill, ON, Canada) at $-80^{\circ} \mathrm{C}$ until use.

Antimicrobial susceptibility testing: Antimicrobial susceptibility tests were performed using seven antibiotics commonly selected in Japanese veterinary dermatological practice: cefalexin (CEX), amoxicillin/clavulanic acid (CVA/AMPC), minocycline (MINO), ofloxacin (OFLX), norfloxacin (NFLX), lincomycin (LCM) and clindamycin (CLDM). All specimens were tested by the disc diffusion method, and classified as susceptible, intermediate or resistant following the Clinical and Laboratory Standards Institute (CLSI) guidelines [5].

DNA extraction: DNA extracts were obtained using QuickGene Mini80 system (Fujifilm Co., Tokyo, Japan) and QuickGene DNA Tissue Kit S (Fujifilm Co.) according to the manufacturer's recommendations.

Species identification using multiplex-polymerase chain reaction (M-PCR) method: M-PCR method for the identification of CoPS species was used in the present study as previously described [32]. DNA fragments were analyzed by electrophoresis in $0.5 \times \mathrm{TBE}$ buffer on a $1.5 \%$ agarose gel stained with $0.5 \mu \mathrm{g} / \mathrm{m} l$ ethidium bromide. To evaluate the sensitivity and specificity, we performed the M-PCR method against type strains of six CoPS species and S. epidermidis, a coagulase-negative staphylococcal species, as a negative control (listed in Table 1). We used these type strains obtained from Gifu Type Culture Collection in the Graduate School of Medicine, Department of Microbiology Regeneration and Advanced Medical Science, Gifu University (Gifu, Japan).

Determination of methicillin resistance by detection of mecA gene: Primers for mecA PCRs, mA1 (5' TGCTATCCACCCTCAAACAGG 3') and mA2 (5' AACGTTGTAACCACCCCAAGA 3') were used in the present study as previously described [27]. The PCR amplifications consisted of pre-denaturing $\left(94^{\circ} \mathrm{C}\right.$ for $\left.2 \mathrm{~min}\right)$, and 30 cycles of denaturation $\left(94^{\circ} \mathrm{C}\right.$ for $\left.1 \mathrm{~min}\right)$, annealing $\left(60^{\circ} \mathrm{C}\right.$ for $\left.1 \mathrm{~min}\right)$

Table 1. Gifu Type Culture collection numbers of staphylococcal type strains used as quality controls in multiplexPCR

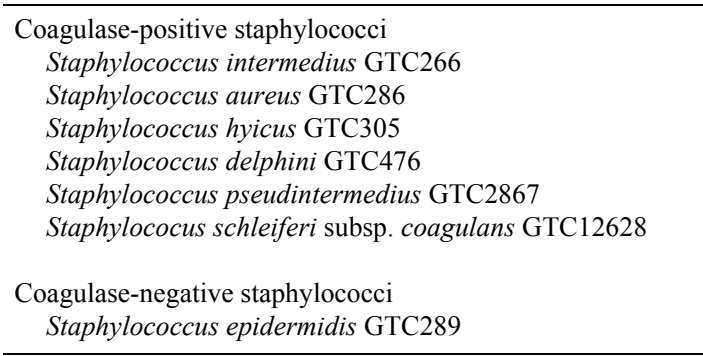

and extension $\left(72^{\circ} \mathrm{C}\right.$ for $\left.1 \mathrm{~min}\right)$, followed by a final extension ( 2 min). Electrophoretic analysis of PCR products (286 bp) was performed as described above. Strains that were $m e c A$-positive or -negative were determined as methicillinresistant (MR) or methicillin-susceptible (MS) strains, respectively.

Statistical analysis: Fisher's exact test was used to compare the prevalence of susceptibility to each antimicrobial between MR and MSCoPS strains. Differences were considered significant for $P<0.05$.

\section{RESULTS}

Isolation and species identification of coagulase-positive staphylococci: One hundred and ninety CoPS were isolated from lesional skin of 186 dogs with pyoderma. Two CoPS species were identified by M-PCR in this study: 170 of $S$. pseudintermedius $(89.5 \%)$ and 20 of S. schleiferi subsp. coagulans (10.5\%). Other CoPS species such as $S$. intermedius and $S$. aureus were not isolated.

Antimicrobial susceptibility tests: The sensitivities of $S$. pseudintermedius and S. schleiferi subsp. coagulans isolates to individual antimicrobials are shown in Table 2. Less than $50 \%$ of $S$. pseudintermedius strains were susceptible to CEX, MINO, OFLX, NFLX, LCM and CLDM (1.2$38.2 \%)$. Sensitivity to CVA/AMPC was the highest $(52.4 \%)$. The frequencies of susceptibility to CEX, CVA/ AMPC and MINO among the $S$. schleiferi subsp. coagulans strains were 55,80 and $70 \%$, while 45,45 and $30 \%$ of strains showed sensitivity to OFLX, NFLX and CLDM, respectively. None of the strains was found to be susceptible to LCM.

Prevalence of methicillin-resistant (MR) strains: The levels of prevalence of MR S. pseudintermedius (MRSP) and MR S. schleiferi subsp. coagulans (MRSS) were $66.5 \%$ $(113 / 170)$ and $30.0 \%(6 / 20)$, respectively.

Difference in susceptibility between methicillin-resistant (MR) and methicillin-susceptible (MS) staphylococcal isolates: The proportions of strains susceptible to individual antimicrobials between MR and MSCoPS strains are listed in Table 3. The frequencies of susceptibility to CEX, CVA/ AMPC, OFLX, NFLX and CLDM in MRCoPS strains were significantly lower than those of MSCoPS strains $(P<0.01)$. One of the 71 MSCoPS strains and one of the 119 MRCoPS strains were susceptible to LCM, and there was no significant difference in the susceptibility rates to MINO and LCM between MR and MSCoPS strains.

\section{DISCUSSION}

In the present study, Staphylococcus pseudintermedius was identified in canine pyoderma in $89.5 \%$ of total isolates. Since first reported [8], S. pseudintermedius has been thought to be a staphylococcal species of commensal flora and a major cause of skin infection including pyoderma [1, 31]. The results of this study support the assertion that $S$. pseudintermedius may be a major pathogen of canine pyoderma. 
Table 2. Antimicriobial susceptibility (\%) of CoPS isolated from canine pyoderma

\begin{tabular}{|c|c|c|c|c|c|c|c|c|c|c|c|c|}
\hline \multirow[t]{2}{*}{ Antimicrobials } & \multicolumn{4}{|c|}{$\begin{array}{l}\text { S. pseudintermedius } \\
\mathrm{n}=170\end{array}$} & \multicolumn{4}{|c|}{$\begin{array}{l}\text { S. schleiferi subsp. coagulans } \\
\mathrm{n}=20\end{array}$} & \multicolumn{4}{|c|}{$\begin{array}{c}\text { Total } \\
\mathrm{n}=190\end{array}$} \\
\hline & $\mathrm{S}$ & I & & $\mathrm{R}$ & & $S$ & I & $\mathrm{R}$ & S & I & & $\mathrm{R}$ \\
\hline Cefalexin & $65(38.2)$ & $6(3.5)$ & 99 & $(58.2)$ & 11 & $(55.0)$ & $0(0)$ & $9(45.0)$ & $76(40.0)$ & $6(3.2)$ & 108 & $(56.8)$ \\
\hline Amoxicillin/clavulanic acid & $89(52.4)$ & ND & 81 & (47.6) & 16 & $(80.0)$ & ND & $4(20.0)$ & $105(55.3)$ & ND & 85 & (44.7) \\
\hline Minocycline & $59(34.7)$ & $65(38.2)$ & 46 & $(27.1)$ & 14 & $(70.0)$ & $6(30.0)$ & $0(0)$ & $73(38.4)$ & 71 (37.4) & 46 & (24.2) \\
\hline Ofloxacin & $53(31.2)$ & $10(5.9)$ & 107 & $(62.9)$ & 9 & $(45.0)$ & $2(10.0)$ & $9(45.0)$ & $62(32.6)$ & $11(5.8)$ & 117 & (61.6) \\
\hline Norfloxacin & $58(34.1)$ & $8(4.7)$ & 104 & $(61.2)$ & 9 & $(45.0)$ & $2(10.0)$ & $9(45.0)$ & $67(35.3)$ & $9(4.7)$ & 114 & $(60.0)$ \\
\hline Lincomycin & $2(1.2)$ & $10(5.9)$ & 158 & (92.9) & 0 & (0) & $6(30.0)$ & $14(70.0)$ & $2(1.1)$ & $15(7.9)$ & 173 & (91.1) \\
\hline Clindamycin & $19(11.2)$ & $30(17.6)$ & 121 & $(71.2)$ & 6 & $(30.0)$ & $12(60.0)$ & $2(10.0)$ & $25(13.2)$ & $41(21.6)$ & 124 & $(65.3)$ \\
\hline
\end{tabular}

S: susceptible; I: intermediate; R: resistant, ND: not determined. S, I, and R were classified according to Clinical and Laboratory Standards Institute guidelines.

Table 3. Antimicrobial susceptibility of methicillin-susceptible and -resistant staphylococci isolated from dogs with pyoderma

\begin{tabular}{lcccccc}
\hline \multirow{2}{*}{ Antimicrobials } & \multicolumn{5}{c}{ Number (\%) of susceptible strains } \\
& MSSP & MRSP & MSSS & MRSS & MSCoPS* & MRCoPS* \\
\cline { 2 - 7 } & $(\mathrm{n}=57)$ & $(\mathrm{n}=113)$ & $(\mathrm{n}=14)$ & $(\mathrm{n}=6)$ & $(\mathrm{n}=71)$ & $(\mathrm{n}=119)$ \\
\hline Cefalexin & $42(73.7)$ & $23(20.4)$ & $11(78.6)$ & $0(0)$ & $53(74.6)^{\mathrm{a})}$ & $23(19.3)^{\mathrm{a})}$ \\
Amoxicillin/clavulanic acid & $54(94.7)$ & $35(31.0)$ & $14(100)$ & $2(33.3)$ & $68(95.8)^{\mathrm{a})}$ & $37(31.1)^{\mathrm{a})}$ \\
Minocycline & $20(35.1)$ & $39(34.5)$ & $11(78.6)$ & $3(50.0)$ & $32(45.1)$ & $42(35.3)$ \\
Ofloxacin & $35(61.4)$ & $18(15.9)$ & $8(57.1)$ & $1(16.7)$ & $43(60.6)^{\mathrm{a})}$ & $19(16.0)^{\mathrm{a})}$ \\
Norfloxacin & $39(68.4)$ & $19(16.8)$ & $8(57.1)$ & $1(16.7)$ & $47(66.2)^{\mathrm{a})}$ & $20(16.8)^{\mathrm{a})}$ \\
Lincomycin & $1(1.8)$ & $1(0.9)$ & $0(0)$ & $0(0)$ & $1(1.4)$ & $1(0.8)$ \\
Clindamycin & $10(17.5)$ & $9(8.0)$ & $6(42.9)$ & $0(0)$ & $16(22.5)^{\mathrm{b})}$ & $9(7.6)^{\mathrm{b})}$ \\
\hline
\end{tabular}

MSSP: methicillin-susceptible $S$. pseudintermedius. MRSP: methicillin-resistant S. pseudintermedius. MSSS: methicillinsusceptible S. schleiferi subsp. coagulans. MRSS: methicillin-resistant $S$. schleiferi subsp. coagulans. * MSCoPS and MRCoPS are equal to MSSP plus MSSS and MRSP plus MRSS, respectively. a) Different superscripts within columns indicate statistically significant difference $(P<0.0001)$. b) Different superscripts within columns indicate statistically significant difference $(P<0.01)$.

S. schleiferi subsp. coagulans were isolated with a frequency of $10.5 \%$ from the lesional skin of dogs in the present study. Although the organism was first isolated from external auditory meatus with canine otitis externa [19], recent studies reported that the organism has also been isolated from dogs with pyoderma $[3,12,18]$. Our results also suggest that this organism is the most important pathogen in canine pyoderma after $S$. pseudintermedius.

The frequency of methicillin-resistant $S$. pseudintermedius (MRSP) isolation in this study was markedly higher than that previously observed by other researchers who reported the existence of MRSP isolated from the skin of healthy and affected dogs $[16,29,30]$. All bacterial specimens in this study were collected from dogs in a veterinary teaching hospital and a veterinary dermatology clinic. Therefore, many of the samples were possibly isolated from cases of recurrent pyoderma. Furthermore, some of these cases may have received antimicrobial treatment in the past, although the details remain unclear. Consequently, owing to selective pressure, staphylococcal isolates in this study may have showed a higher than usual rate of methicillin resistance. The prevalence of MR S. aureus (MRSA) strains in human hospitals was found to be $66.8 \%$ in Japan [2]. These results demonstrated a high prevalence of MR strains in humans similar to that we identified in dogs.

The rates of antimicrobial susceptibility to CEX, CVA/
AMPC, OFLX and NFLX in MRCoPS isolates in this study were significantly lower than those in MSCoPS isolates. MR staphylococci produce an altered penicillin-binding protein (PBP 2' or PBP 2a) coded by the mecA gene, that has extremely lower affinity for binding $\beta$-lactams $[17,37]$. Therefore, the PBP 2' makes bacteria resistant to all $\beta$-lactam antibiotics. In contrast, quinolone resistance in staphylococci is caused by mutations in the gyrA, gyrB, grlA or $\operatorname{grl} B$ genes coding DNA gyrase and topoisomerase, that are essential for bacterial DNA synthesis [20,35]. There are some reports that MRSA isolates obtained from human hosts had mutations in these genes and these isolates were resistant against old generation quinolones such as NFLX or ciprofloxacin $[6,26,33]$. Similarly, Descloux et al. reported that all MRSP isolates from diseased dogs also had mutations in these genes together with resistance to enrofloxacin [7]. These reports and our results suggest that it is important to confirm the susceptibility to quinolones for appropriate use in treatment of MRCoPS infections in dogs.

In this study, there was no significant difference in the susceptibility to MINO between MR and MSCoPS isolates. Two mechanisms of resistance to tetracyclines have been identified in staphylococci: active drug efflux mechanism resulting from the acquisition of tet $K$ and tet $L$ genes, and ribosomal protections mediated by tet $M$ or tet $O$ genes [11, $21,28]$. In addition, it was also reported that tetM- or tetK- 
and tetM-positive MRSA isolates had high levels of resistance to MINO [36]. However, several recent publications have reported that in vitro susceptibility of MINO against MRSA isolates from human hosts was significantly high as compared to other antimicrobials such as CLDM, CVA/ AMPC or levofloxacin [9, 34, 40]. In fact, our data also indicate that the proportion of MRCoPS strains resistant to MINO was the lowest among those for seven antimicrobial agents (29.4\% of all MRCoPS isolates, data not shown). In addition, the proportion of MRCoPS isolates that were classified with intermediate susceptibility to MINO accounted for $35.3 \%(42 / 119)$ of the total CoPS isolates in our study (data not shown). The efficacy of MINO against MRCoPS may be revealed by determination of their minimum inhibitory concentrations and identification of the tet genes in addition to our results.

The prevalence of susceptibility to LCM and CLDM was extremely low in both MR and MSCoPS isolates in our study. Similarly, Futagawa-Saito et al. indicated comparably low levels of susceptibility to LCM in staphylococci isolated from healthy (8/44) and diseased (36/44) dogs in Japan [13]. However, the incidence of susceptibility to LCM and CLDM reported by Vanni et al. was quite high in Staphylococcus spp., which were isolated from healthy $(255 / 310)$ and diseased (55/310) dogs in Italy between 2006 and 2007 [39]. These investigations and our results suggest that the levels of susceptibility to LCM in staphylococcal isolates from diseased dogs are possibly lower than those from healthy dogs and, at least in Japan, there is a low frequency of susceptibility to lincosamides such as LCM and CLDM in staphylococcal isolates from diseased dogs, and Japanese veterinary clinicians should not expect therapeutic efficacy of lincosamides against canine pyoderma.

In conclusion, our results indicate a high level of resistance of CoPS isolates to the antimicrobials usually used for treatment against staphylococcal infections. Moreover, a high level of MRCoPS strains seems to be increasingly common in cases of canine pyoderma. However, there are significant differences in antimicrobial susceptibilities between staphylococcal species, and between MSCoPS and MRCoPS strains, especially for $\beta$-lactams and quinolones. This suggests the importance of performing antimicrobial susceptibility testing and confirming the presence of methicillin resistance among staphylococcal isolates from canine pyoderma.

ACKNOWLEDGMENTS. This work was supported by a Grant-in-Aid for Scientific Research from the Ministry of Education, Science, Sports and Culture, and Special Coordination Funds for Promoting Science and Technology of the Science and Technology Agency of the Japanese Government.

\section{REFERENCES}

1. Bannoehr, J., Ben Zakour, N. L., Waller, A. S., Guardabassi, L., Thoday, K. L., van den Broek, A. H. and Fitzgerald, J. R.
2007. Population genetic structure of the Staphylococcus intermedius group: insights into agr diversification and the emergence of methicillin-resistant strains. J. Bacteriol. 189: 86858692.

2. Bell, J. M. and Turnidge, J. D. 2002. High prevalence of oxacillin-resistant Staphylococcus aureus isolates from hospitalized patients in Asia-Pacific and South Africa: results from SENTRY antimicrobial surveillance program, 1998-1999. Antimicrob. Agents Chemother. 46: 879-881.

3. Bes, M., Guerin-Faublee, V., Freney, J. and Etienne, J. 2002. Isolation of Staphylococcus schleiferi subspecies coagulans from two cases of canine pyoderma. Vet. Rec. 150: 487-488.

4. Biberstein, E. L., Jang, S. S. and Hirsh, D. C. 1984. Species distribution of coagulase-positive staphylococci in animals. $J$. Clin. Microbiol. 19: 610-615.

5. Clinical and Laboratory Standards Institute. 2009. Performance Standards for Antimicrobial Susceptibility Testing; Nineteenth Informational Supplement, M100-S19., CLSI, Wayne.

6. Coskun-Ari, F. F. and Bosgelmez-Tinaz, G. 2008. grlA and gyrA mutations and antimicrobial susceptibility in clinical isolates of ciprofloxacin- methicillin-resistant Staphylococcus aureus. Eur. J. Med. Res. 13: 366-370.

7. Descloux, S., Rossano, A. and Perreten, V. 2008. Characterization of new staphylococcal cassette chromosome mec (SCC$m e c)$ and topoisomerase genes in fluoroquinolone- and methicillin-resistant Staphylococcus pseudintermedius. J. Clin. Microbiol. 46: 1818-1823.

8. Devriese, L. A., Vancanneyt, M., Baele, M., Vaneechoutte, M., De Graef, E., Snauwaert, C., Cleenwerck, I., Dawyndt, P., Swings, J., Decostere, A. and Haesebrouck, F. 2005. Staphylococcus pseudintermedius sp. nov., a coagulase-positive species from animals. Int. J. Syst .Evol. Microbiol. 55: 1569-1573.

9. Draghi, D. C., Tench, S., Dowzicky, M. J. and Sahm, D. F. 2008. Baseline in vitro activity of tigecycline among key bacterial pathogens exhibiting multidrug resistance. Chemotherapy 54: $91-100$.

10. Fazakerley, J., Nuttall, T., Sales, D., Schmidt, V., Carter, S. D., Hart, C. A. and McEwan, N. A. 2009. Staphylococcal colonization of mucosal and lesional skin sites in atopic and healthy dogs. Vet. Dermatol. 20: 179-184.

11. Fluit, A. C., Florijn, A., Verhoef, J. and Milatovic, D. 2005. Presence of tetracycline resistance determinants and susceptibility to tigecycline and minocycline. Antimicrob. Agents Chemother. 49: 1636-1638.

12. Frank, L. A., Kania, S. A., Hnilica, K. A., Wilkes, R. P. and Bemis, D. A. 2003. Isolation of Staphylococcus schleiferi from dogs with pyoderma. J. Am. Vet. Med. Assoc. 222: 451-454.

13. Futagawa-Saito, K., Ba-Thein, W. and Fukuyasu, T. 2007. High occurrence of multi-antimicrobial resistance in Staphylococcus intermedius isolates from healthy and diseased dogs and domesticated pigeons. Res. Vet. Sci. 83: 336-339.

14. Griffeth, G. C., Morris, D. O., Abraham, J. L., Shofer, F. S. and Rankin, S. C. 2008. Screening for skin carriage of methicillinresistant coagulase-positive staphylococci and Staphylococcus schleiferi in dogs with healthy and inflamed skin. Vet. Dermatol. 19: 142-149.

15. Gross, T. A., Ihrke, P. J., Walder, E. J. and Affolter, V. K. 2005. Pustular diseases of the epidermis. pp. 6-9. In: Skin Diseases of the Dog and Cat: Clinical and Histopathologic Diagnosis, 2nd ed., Blackwell Science Ltd., Oxford.

16. Hanselman, B. A., Kruth, S. and Weese, J. S. 2008. Methicillin-resistant staphylococcal colonization in dogs entering a veterinary teaching hospital. Vet. Microbiol. 126: 277-281. 
17. Hartman, B. J. and Tomasz, A. 1984. Low-affinity penicillinbinding protein associated with beta-lactam resistance in Staphylococcus aureus. J. Bacteriol. 158: 513-516.

18. Holm, B. R., Petersson, U., Morner, A., Bergstrom, K., Franklin, A. and Greko, C. 2002. Antimicrobial resistance in staphylococci from canine pyoderma: a prospective study of firsttime and recurrent cases in Sweden. Vet. Rec. 151: 600-605.

19. Igimi, S., Takahashi, E. and Mitsuoka, T. 1990. Staphylococcus schleiferi subsp. coagulans subsp. nov., isolated from the external auditory meatus of dogs with external ear otitis. Int. J. Syst. Bacteriol. 40: 409-411.

20. Ito, H., Yoshida, H., Bogaki-Shonai, M., Niga, T., Hattori, H. and Nakamura, S. 1994. Quinolone resistance mutations in the DNA gyrase gyrA and gyrB genes of Staphylococcus aureus. Antimicrob. Agents Chemother. 38: 2014-2023.

21. Jones, C. H., Tuckman, M., Howe, A. Y., Orlowski, M., Mullen, S., Chan, K. and Bradford, P. A. 2006. Diagnostic PCR analysis of the occurrence of methicillin and tetracycline resistance genes among Staphylococcus aureus isolates from phase 3 clinical trials of tigecycline for complicated skin and skin structure infections. Antimicrob. Agents Chemother. 50: 505-510.

22. Jones, R. D., Kania, S. A., Rohrbach, B. W., Frank, L. A. and Bemis, D. A. 2007. Prevalence of oxacillin- and multidrugresistant staphylococci in clinical samples from dogs: 1,772 samples (2001-2005). J. Am. Vet. Med. Assoc. 230: 221-227.

23. Kania, S. A., Williamson, N. L., Frank, L. A., Wilkes, R. P., Jones, R. D. and Bemis, D. A. 2004. Methicillin resistance of staphylococci isolated from the skin of dogs with pyoderma. Am. J. Vet. Res. 65: 1265-1268.

24. Kim, T. J., Na, Y. R. and Lee, J. I. 2005. Investigations into the basis of chloramphenicol and tetracycline resistance in Staphylococcus intermedius isolates from cases of pyoderma in dogs. J. Vet. Med. B. Infect. Dis. Vet. Public Health 52: 119-124.

25. Mason, I. S. and Kietzmann, M. 1999. Cephalosporins-pharmacological basis of clinical use in veterinary dermatology. Vet. Dermatol. 10: 187-192.

26. Noguchi, N., Okihara, T., Namiki, Y., Kumaki, Y., Yamanaka, Y., Koyama, M., Wakasugi, K. and Sasatsu, M. 2005. Susceptibility and resistance genes to fluoroquinolones in methicillinresistant Staphylococcus aureus isolated in 2002. Int. J. Antimicrob. Agents 25: 374-379.

27. Okuma, K., Iwakawa, K., Turnidge, J. D., Grubb, W. B., Bell, J. M., O’Brien, F. G., Coombs, G. W., Pearman, J. W., Tenover, F. C., Kapi, M., Tiensasitorn, C., Ito, T. and Hiramatsu, K. 2002. Dissemination of new methicillin-resistant Staphylococcus aureus clones in the community. J. Clin. Microbiol. 40: 4289-4294.

28. Petersen, P. J., Jacobus, N. V., Weiss, W. J., Sum, P. E. and Testa, R. T. 1999. In vitro and in vivo antibacterial activities of a novel glycylcycline, the 9-t-butylglycylamido derivative of minocycline (GAR-936). Antimicrob. Agents Chemother. 43: 738-744.

29. Ruscher, C., Lubke-Becker, A., Wleklinski, C. G., Soba, A., Wieler, L. H. and Walther, B. 2009. Prevalence of Methicillinresistant Staphylococcus pseudintermedius isolated from clini- cal samples of companion animals and equidaes. Vet. Microbiol. 136: 197-201.

30. Sasaki, T., Kikuchi, K., Tanaka, Y., Takahashi, N., Kamata, S. and Hiramatsu, K. 2007. Methicillin-resistant Staphylococcus pseudintermedius in a veterinary teaching hospital. J. Clin. Microbiol. 45: 1118-1125.

31. Sasaki, T., Kikuchi, K., Tanaka, Y., Takahashi, N., Kamata, S. and Hiramatsu, K. 2007. Reclassification of phenotypically identified Staphylococcus intermedius strains. J. Clin. Microbiol. 45: 2770-2778.

32. Sasaki, T., Tsubakishita, S., Tanaka, Y., Sakusabe, A., Ohtsuka, M., Hirotaki, S., Kawakami, T., Fukata, T. and Hiramatsu, K. 2010. Multiplex-PCR method for species identification of coagulase-positive staphylococci. J. Clin. Microbiol. 48: 765-769.

33. Schmitz, F. J., Jones, M. E., Hofmann, B., Hansen, B., Scheuring, S., Luckefahr, M., Fluit, A., Verhoef, J., Hadding, U., Heinz, H. P. and Kohrer, K. 1998. Characterization of grlA, grlB, gyrA, and gyrB mutations in 116 unrelated isolates of Staphylococcus aureus and effects of mutations on ciprofloxacin MIC. Antimicrob. Agents Chemother. 42: 1249-1252.

34. Souli, M., Kontopidou, F. V., Koratzanis, E., Antoniadou, A., Giannitsioti, E., Evangelopoulou, P., Kannavaki, S. and Giamarellou, H. 2006. In vitro activity of tigecycline against multiple-drug-resistant, including pan-resistant, gram-negative and gram-positive clinical isolates from Greek hospitals. Antimicrob. Agents Chemother. 50: 3166-3169.

35. Tokue, Y., Sugano, K., Saito, D., Noda, T., Ohkura, H., Shimosato, Y. and Sekiya, T. 1994. Detection of novel mutations in the gyrA gene of Staphylococcus aureus by nonradioisotopic single-strand conformation polymorphism analysis and direct DNA sequencing. Antimicrob. Agents Chemother. 38: 428431.

36. Trzcinski, K., Cooper, B. S., Hryniewicz, W. and Dowson, C. G. 2000. Expression of resistance to tetracyclines in strains of methicillin-resistant Staphylococcus aureus. J. Antimicrob. Chemother. 45: 763-770.

37. Utsui, Y. and Yokota, T. 1985. Role of an altered penicillinbinding protein in methicillin- and cephem-resistant Staphylococcus aureus. Antimicrob. Agents Chemother. 28: 397-403.

38. Van Hoovels, L., Vankeerberghen, A., Boel, A., Van Vaerenbergh, K. and De Beenhouwer, H. 2006. First case of Staphylococcus pseudintermedius infection in a human. J. Clin. Microbiol. 44: 4609-4612.

39. Vanni, M., Tognetti, R., Pretti, C., Crema, F., Soldani, G., Meucci, V. and Intorre, L. 2009. Antimicrobial susceptibility of Staphylococcus intermedius and Staphylococcus schleiferi isolated from dogs. Res. Vet. Sci. 87: 192-195.

40. Waites, K. B., Duffy, L. B. and Dowzicky, M. J. 2006. Antimicrobial susceptibility among pathogens collected from hospitalized patients in the United States and in vitro activity of tigecycline, a new glycylcycline antimicrobial. Antimicrob. Agents Chemother. 50: 3479-3484.

41. Weese, J. S. and van Duijkeren, E. 2010. Methicillin-resistant Staphylococcus aureus and Staphylococcus pseudintermedius in veterinary medicine. Vet. Microbiol. 140: 418-429. 